\title{
DESPLIEGUES DE UNA COMPLICACIÒN: SOBRE LA TÉCNICA, LA NATURALEZA Y LO
} HUMANO

\author{
Víctor Veloso Luarte ${ }^{1}$ \\ Vicente Valle Ureta $^{2}$
}

\section{Resumen/Abstract}

En lo que sigue, pretendemos desplegar una tensión ya instalada en la actualidad que atañe al lugar que los conceptos de "técnica", de "naturaleza" y de "lo humano" vienen a ocupar. En la búsqueda por calibrar esta triple relación revisamos algunos rasgos que consideramos centrales para esta cuestión, en las filosofías de Bernard Stiegler, Deleuze y Guattari, y Jean-Luc Nancy. A la luz de estas propuestas subrayamos convergencias, divergencias, y proyecciones en vistas a sostener el carácter problemático, y precisamente por ello urgente, que presenta la técnica en la época contemporánea. Tramada con y en la naturaleza, y desplazando el lugar central que lo humano ocupó en torno a la técnica, se estremece el tabique que separaba radicalmente a naturaleza y técnica, dejando al humano conmocionado, errante y vagabundo.

Palabras Clave: técnica, naturaleza, humanismo, capitalismo, ecotecnia, maquinismo

\section{DEPLOYMENTS OF A COMPLICATION: ON TECHNIQUE, NATURE AND THE HUMAN}

We intend to unfold an already existing tension among the concepts of technique, nature, and the human. Seeking to gauge this three-fold relationship, we examine what we consider key features in the works of Bernard Stiegler, Deleuze \& Guattari, and Jean-Luc Nancy. We situate ourselves within these authors' philosophical proposals, in order to highlight points in which they converge or diverge, as well as ways in which they help us the project and uphold the problematic and thus urgent, matter of technique in contemporary times. Intertwined with/in nature, the place of the human is being currently displaced from the central role it once occupied around technique, destabilizing the partition that once radically separated nature and technique, leaving humans bewildered, errant, and wandering.

Keywords: technique, nature, humanities, capitalism, eco-technique, mechanism

1 Chileno, Universidad de Chile. E-mail: victor.veloso@ug.uchile.cl

2 Chileno, Universidad de Chile. E-mail: vicente.valle@ug.uchile.cl 


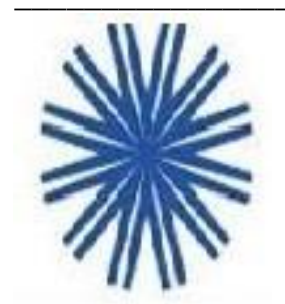

\section{Cómo comenzar}

En lo que sigue, pretendemos desplegar una tensión ya instalada en la actualidad, que atañe al lugar que los conceptos de "técnica", de "naturaleza" y de "lo humano" vienen a ocupar. En la búsqueda por calibrar esta triple relación, revisamos algunos rasgos que consideramos centrales, para esta cuestión, en las filosofías de Bernard Stiegler, Deleuze y Guattari, y Jean-Luc Nancy, destacando sus proyecciones, convergencias y divergencias. Finalmente, sopesamos el recorrido a fin de construir antes una interrogante que una hipótesis, o incluso, si cabe, una interrogante como hipótesis.

Partimos, entonces, del presentimiento de una pregunta a cuya formulación quisiéramos 1legar. Esta interrogante involucrará la técnica, por un lado; la naturaleza, por otro; y lo que se ha dado en llamar lo humano, aparente o tradicional bisagra entre ambos términos, constituyendo ya otro punto. Cada idea aparece suficientemente grande, inabarcable, por lo que la articulación de estos tres polos es, ahora, el único recurso para modularlos, para acotarlos y verlos ceñirse el uno a los otros, incluso el uno en los otros. No obstante, esta modulación cuenta de antemano con una situación, un estar situados: esta pregunta la queremos plantear hoy.

¿Qué tiempo, en torno a esta triada, designa ese "hoy”? ¡Bastaría revisar los periódicos, las noticias de hoy! Sabemos, sin embargo, que al ensayar un pensamiento, "hoy" abarca un bloque más vasto que el luminoso "ahora", la vastedad de sus sombras, y que el hecho de preguntar hoy algo sobre el hoy, es hacer señas a otros tiempos a través de esas sombras: a un pasado respecto del cual hoy difiere, haciéndolo reconocible, con lo que nos preguntamos por esa diferencia respecto de lo que fue antes que hoy; o a un futuro respecto del cual el hoy diferirá, haciendo de este hoy un recurso para pensar el mañana, con lo que nos preguntamos por la posibilidad de esa diferencia por venir. Hacia ambos extremos, los límites del hoy se hacen difusos. ¿Cómo hacerse de las fórmulas, más o menos exactas, para situar hoy la cuestión de la técnica, la naturaleza y lo humano?

Si se nos permite, quisiéramos partir por señalar, acaso recordar, que este hoy hunde sus raíces en un ayer no tan lejano: los procesos que dan pie a la modernidad redefinen las relaciones entre la técnica, la naturaleza y la humanidad. Rápidamente: las revoluciones industriales permiten dar un salto cualitativo a las técnicas, modificando el carácter vivencial del tiempo y el espacio con transformaciones en la 
comunicación y el transporte, acelerando además, agudamente, la producción; la naturaleza deviene entonces, se ha señalado bastante ya, un elemento a disposición de la técnica, de manera que nos provee de energías, de materias primas, de explanadas para la habitación humana, en síntesis, y en todo sentido, la naturaleza deviene un recurso; el ser humano, como sujeto, se lograría distinguir ontológicamente del resto de la naturaleza, objeto al cual puede medir, comprender, predecir y calcular. Esto se ve acompañado por procesos de secularización que destronan lo divino para ocupar ese vacío, aún si pobremente, con la subjetividad, dato o proyecto sin dudas nuclear en este periodo.

Así, lo propiamente humano, tras diversos eventos -incluida la conquista de América-, se vuelve objeto del saber secular humano, de las ciencias humanas. Se ha inventado un mundo, nuevo, cuya promesa política es la libertad y cuyo anhelo tecno-científico y económico es el progreso, esperanzas que tomarán, en cada caso concreto, diversas e incluso contradictorias formas.

De pronto, hacia el siglo XX, tiene lugar un acontecimiento que ¿se podrá decir así? remata esta historia: dos bombas atómicas caen en Japón, en el marco de una guerra de alcance mundial, dando cuenta de que la libertad humana tiene incluso la posibilidad del autoexterminio, cuya condición, desde luego, ha sido tecno-científica, y cuyo objetivo hace parte de una compleja trama de reparto económico del mundo. Hoy esta historia retorna aunque pareciendo menos horrorosa, ocultando el alcance de su desastre, y su nombre, en principio, es antropoceno.

El antropoceno opera como una hipótesis antes que una certeza -y de hecho ya ha dado lugar a discusiones en torno a si no sería mejor denominar este "periodo" como capitaloceno, o, siguiendo a Donna Haraway (2016), chthuluceno, de manera que no quisiéramos inscribir nuestra pregunta en ese marco. Más bien, la pregunta que intuimos interroga qué sea ese marco: ¿antropoceno o tecnoceno como catástrofe, chthuluceno u otra época por venir?

En cualquier caso, lo indicado por esos nombres, así como todo el siglo XX, dan muestras de lo que queremos señalar: hoy la relación entre la técnica, la naturaleza y lo humano, difieren fuertemente de la relación que pudieron establecer en otros momentos de la historia humana -incluso de la historia naturalpero más aún, y sobre todo, el hoy difiere incluso de ese cercano ayer al que aún se arraiga el hoy: las promesas de libertad se ven puestas en cuestión, y el saber tecno-científico deviene ambiguo y amorfo, 
entre una creciente mala fama y su aún estable carácter de religión secular, con lo que ya no ofrece solo progreso: surgen enfermedades incurables, la contaminación figura como efecto indeseado e inevitable en términos absolutos, el riesgo hace parte de cualquier cálculo racional de progreso, crecen la apatía y el aislamiento social, junto a nuevos espacios, digitales, para el crimen y la perversión. Acaso esta sea la consecución de los objetivos, de los fines de la modernidad; acaso sea su agotamiento, proceso de acercamiento, a la vez tan moderno mas asintótico, al fin de la modernidad; acaso se haya abierto una brecha, aun invisible, hacia otra época, a un fin de la modernidad y su después. Pero no quisiéramos evaluar qué se juega en este fin: la modernidad realizada, agotada o acabada, o incluso si algo de la modernidad sería lo que se perdió pero reorientará nuestros pasos. Nuestra pregunta, vemos ahora, es más humilde, más acotada: tan solo se pregunta por la técnica, la naturaleza y lo humano, hoy.

\section{Técnica como hominización}

Y así, en un triple vaivén en torno al fin y un triple vaivén en torno a la triada naturaleza-técnicahumanidad, erramos oscilantes, como con el equilibrio de una navegación tímida que no atisba costas ni puertos. Y entonces nos encontramos, impulsadas nuestras velas por un buen ventarrón, con la lectura de ciertas filosofías de la técnica que, rehuyendo a una antropología, incurrían de todos modos en hipótesis antropogenéticas, en una suerte de hominización no humanista. Puede que aquí haya, entonces, una relación entre los componentes de esta, nuestra triada interrogante, y puede que haya alguna referencia al hoy.

Es Bernard Stiegler quien señala este camino, intentando una síntesis de diversas corrientes. Siguiendo a André Leroi-Gourhan, Stiegler (1994) dirá que la hominización comienza por los pies, o más exactamente, por la liberación de las manos, que las abre hacia la tecnicidad. Al revés, podríamos decir, como lo planteará luego este autor, que la técnica es la que constituye lo humano, en el seno de la naturaleza. La técnica es la invención del ser humano, frase que se debe leer poniendo en duda quién es sujeto y quien predicado de la oración: se trata de una exteriorización, en herramientas, que inventa a lo humano. Vale decir, lo humano no precede, sino que sucede a la técnica. Pero, más concretamente, ¿qué es lo que la técnica, entonces, exterioriza? Stiegler dirá que es una memoria: un saber. Pero falta subrayar un detalle fundamental a este argumento: la exteriorización que refiere Stiegler no exterioriza una interioridad, una memoria ya habida y ya humana, sino que la crea precisamente en esa exteriorización, y la crea como humana. De manera que "exteriorizar" no nombra un llevar hacia afuera lo interior, sino un movimiento 
creativo antes que expresivo: exteriorizar es darle soporte a lo humano que solo emergerá allí -incluso, como veremos luego, cierto adelantamiento de la técnica respecto de lo humano y lo social-, es espacializar flujos vitales y temporales en órganos o artificios sensoriales, que cortan estas funciones del pensamiento, la sensación y el movimiento antes animal, para producir los discretos aparatos y objetos que soportarán lo humano.

Esta hipótesis antropogenética de la exteriorización tiene una ventaja que cabe subrayar: pareciera incorporar a la técnica como un gesto de y en la naturaleza, una transformación de la vida "por otros medios diferentes de la vida" (Stiegler, 1994, p. 80), de modo que la técnica es una expresión de la vida, una naturaleza-extra-biológica y no una prótesis ni una herramienta al servicio de lo humano ya constituido y esencializado. No es que la naturaleza sea técnica, sino que puede la técnica, tiene la capacidad o la potencia de producirla desde sí, y la prueba de ello es lo humano, naturalmente técnico. De este modo, la técnica no surge, en primer lugar, como algo que se opone a la naturaleza, ni tampoco lo humano ocupa un rango axiológicamente distinto del de la naturaleza. El nodo que reúne los tres términos sobre los que aquí llamamos la atención es la técnica.

Ya comprendida la hipótesis de la exteriorización, hace falta ver cómo se explican las tensiones que se hacen lugar hoy, en este marco, en lo que para Stiegler (2016) será un diagnóstico de proletarización, esto es, como el despojo del ser humano de sus propiedades, que lo deja abandonado a su pura prole, argumento que Stiegler extiende más allá de la expropiación de medios productivos hasta una expropiación técnica de sus saberes y capacidades. El nodo, decíamos, es la técnica, exteriorización de un saber, constitución de lo humano. Es este movimiento el que para Stiegler constituye la fuente de todos los problemas. Como decíamos, la técnica, como exteriorización, produce lo humano en un soporte que "contiene" saber. Vale decir, el saber del ser humano, y supuestamente a diferencia de otros animales, está fuera de él, o sea, su saber es objeto a su alcance antes que característica, propiedad, talento o instinto inherente a él. Hay, así, un saber-hacer: desde las primeras herramientas hasta el maquinismo industrial, el hacer del ser humano es puesto en objetos que exterioriza esa actividad. Así, el ser humano no necesita saber-hacer las cosas que la máquina sabe hacer. Esto se puede ejemplificar en la proletarización que en el siglo XIX ve Marx.

Más contemporáneamente, el consumismo operaría un nuevo nivel de exteriorización de un saber, esta vez, del saber-vivir, del que el ser humano puede prescindir una vez que, antes que vérselas con la práctica 
en el mundo, solo requiere de un poder de compra. Esto recuerda a la película Wall-E donde, como sostiene Mark Fisher (2019), Pixar gasta dinero como ninguna otra corporación en insultar a su público, presentando la imagen de humanos como consumidores obesos, infantilizados, atados a sus asientos viendo una pantalla y bebiendo a sorbos las tonterías que hay en sus tazones, viendo completamente atrofiada su movilidad.

Pero todo esto no se agotaría en eso. Para Stiegler (2014), el siglo XXI supondría una nueva proletarización, ya no solo del saber-hacer ni el saber-vivir, sino del saber-teorizar: en menos de treinta años se salió de un escenario industrial que duró dos siglos, creando nuevas tecnologías digitales que nos permiten tener acceso al tiempo-luz, casi a la instantaneidad, cortocircuitando toda posibilidad de pensar lo que está sucediendo. Stiegler (2014), siguiendo la tesis de Chris Anderson, afirmará que nuestro tiempo, el de los big data, anunciaría el "fin de la teoría" (Stiegler 2014: 151-152).

Estos tres niveles de proletarización son, al parecer de Stiegler, inherentes a la tecnicidad que constituye a lo humano y el problema radicaría en la acelerada evolución de los dispositivos técnicos que van dejando atrás a los seres humanos, dificultando lo que el autor, siguiendo a Simondon (2015), denomina individuación y que entendemos como los procesos mediante los que los vivientes logran resolver tensiones respecto de su entorno, procesos que transforman también al individuo, constituyendo modificaciones de todo el sistema de relaciones entre individuo y medio. De esta manera, la proletarización es el abandono del ser humano a una relación difícil, irresuelta, con su entorno, un empobrecimiento de lo humano o una producción de lo humano en tanto que empobrecimiento.

Pero Stiegler se piensa antes como un realista que como un pesimista, de manera que hay una terapéutica positiva contra la proletarización, que radicaría en que el ser humano pueda evolucionar con su medio asociado abriéndose a una experiencia creativa de producción, operación que requiere de toda una crítica -presente en el trabajo de Stiegler- de la acumulación de capital. No entraremos en este elemento de su pensamiento, pese a que reconocemos su importancia, para preferir destacar antes algo que nos complica en esta hipótesis.

Si la técnica es entendida siempre ya como exteriorización de lo humano mediante discretización de funciones pre-humanas, hay un problema. La hominización parece ocurrirle a una suerte de animal pre- 
humano, cuyas funciones psicomotrices son entonces un continuo respecto de él, "son" él, y que tras discretizarse en un soporte exterior, lo hominizan, liberándolo a tareas más complejas, a la vez que restándole de las ya alcanzadas -que no necesita incorporar, sino tener a la mano-, y así, congelando gestos expropiados, capturados, sustraídos a la transformación, con lo que su propio devenir o su individuación, se ve atropellada. Esto será presentado como la precedencia de la tecnogénesis por sobre una ralentizada sociogénesis. Si cierta reflexividad sobre este proceso puede desandar la aceleración de la técnica respecto de lo humano, es decir, desafiar la alienación de lo humano en la técnica, no anula la duda sobre si este carácter ambiguo del pharmakon técnico, entre remedio y veneno, no tiene como su mínimo común denominador ya la alienación, esta vez lenta, y la sustracción del saber. En otras palabras, la terapéutica positiva contra el pharmakon como veneno no parece dejar de ceñirse a la tecnicidad como hominización-ya-siempre-alienante.

No nos parece trivial que Stiegler recurra a Simondon para ilustrar su problema: la tecnogénesis, adelantada a la sociogénesis ${ }^{3}$, resulta en una alienación que traba los procesos de individuación, y si bien hay una terapéutica para hacer frente a este problema entendido como proletarización, queda planteada y subrayada en su obra el carácter de pharmakon de toda técnica ${ }^{4}$. No es así la cuestión para Simondon (2007). Para este último pensar la técnica es comprender un gesto humano fijado y cristalizado (Simondon, 2007: 34) y la alienación viene de no comprender a la máquina, lo cual redunda en no comprender una parte de lo humano y su individuación. Así, su propósito es reformar la cultura para incorporar a ella esquemas técnicos, para realizar una convergencia entre actividades humanas religiosas, estéticas, científicas y técnicas, y en dicha convergencia la técnica puede mostrarse de otro modo que la alienación, por ejemplo: "El objeto técnico es bello cuando encontró un fondo que le cuaja, del cual quizás sea él la propia figura, es decir, cuando alcanza y expresa el mundo" (Simondon 2007: 203), es decir, lejos de la alienación como connatural a la técnica, ella puede volverse un punto-clave que expresa al viviente en su convergencia con su mundo. El problema, en fin, es que para Stiegler la técnica es pharmakon, y hasta la mejor de las terapéuticas conlleva alienación porque la técnica es alienación al implicar que la tecnogénesis se adelante a la sociogénesis, dificultando en sí misma la individuación humana. En cambio,

3 De acuerdo con Stiegler (2012) se debe comprender “'adelanto' de las identidades técnico-objetivas sobre la identidad psicosocial, (...) [como] un adelanto de la técnica sobre la sociedad" (137-138)

4 Stiegler, siguiendo al Derrida (1996) de La Farmacia de Platón, subraya la ambigüedad del pharmakon como la ambigüedad de toda técnica: la imposibilidad de hacer una distinción que se pretenda exacta entre veneno y cura. Así, la técnica, a la vez que viene como una potencia, viene como una impotencia. 
para Simondon, el desapego de la técnica respecto de la cultura, esto es, de su subordinación a despliegues no técnicos -es decir, no relativos a esta fijación del gesto humano, como lo es la subordinación de la técnica al mercado- hace de la técnica una cuestión abstracta, y entonces el ser humano se ve alienado respecto de su propia actividad: se extraña de su propio gesto.

Así, si bien con Stiegler la relación entre los términos ha cambiado, refrescándonos al descentrar el rol de lo humano entre lo existente, no nos sentimos cómodos aún para ensayar una pregunta sobre lo técnico, lo natural y lo humano, toda vez que se ha incurrido en lo que podríamos denominar un tecnocentrismo, un desplazamiento del humanismo antropocéntrico a la vez que un re-establecimiento de la singularidad humana, esta vez subordinada a la técnica como su concepto íntimo y nuclear. Este tecnocentrismo, creando lo humano desde la naturaleza, opera necesariamente como una suerte de alienación, si bien a veces más feliz, y en este movimiento deja pasar un corte ontológico sutil, incluso blando, que hace saltar a la técnica respecto del resto de los elementos y define siempre a lo humano, efecto de la técnica, como ya alienado. ¿Cómo preguntarnos algo si ya sabríamos qué esperar de la técnica? Si para Stiegler, y según el mismo refiere, la generalización de los teléfonos celulares haría que traspasemos nuestra memoria a las máquinas al punto de que ya no sabemos los números telefónicos de gente muy cercana, nosotros preguntamos: ¿y no nos posibilita esa exteriorización de memoria su prolongación en magnitud y tiempo? ¿No sería pertinente preguntarnos qué gesto y qué mundo se expresan o no allí? Si la generalización de los correctores ortográficos haría temer el fin de la conciencia ortográfica, ¿no es útil recordar que, de todas formas, esta conciencia ha mutado ya desde siempre, no será más bien que nace una nueva conciencia ortográfica, exteriorizada y no por eso ajena a la transformación? ¿Por qué la técnica y su exteriorización de lo humano, promoviendo las transformaciones más allá de lo que ciertos pensamientos quieren conservar como lo humano, traerían solo catástrofe? ¿Por qué solo la reificación de una esencia de lo humano pareciera ser la vara que mida toda seguridad en el mundo y la historia? No es que desconozcamos el problema que plantea Stiegler, ni su gravedad, sino que nos resistimos a que esta antropogénesis tecnocéntrica no ofrezca más que alienación.

Por otro lado, la hominización tecnocéntrica que aquí se plantea insiste en una ligazón unívoca entre humanos y técnica, dejando de lado todo un repertorio de técnicas no humanas: los nidos de las aves, las colmenas de las abejas, las herramientas de chimpancés y nutrias. ¿Se nos dirá que ya habíamos resuelto hace tanto, modernos y occidentales como somos, que la diferencia con los artefactos humanos es su 
proyección consciente, calculante e histórico-cultural? Responderemos que Stiegler, al invertir esa relación entre humanidad y técnica, abre esta brecha que no quisiéramos abandonar, cabe entonces revisar, profundizar este punto.

\section{Natura sive Machina}

Ha habido lugar para otras hipótesis que aglutinan estas tres ideas, la técnica, la naturaleza y lo humano. Esta vez se tiende a cierta complicación de la distinción entre técnica y naturaleza dejando lo humano en una posición inestable: ciertamente no como incógnita, pero en una situación que cabría revisar. Esto se verá luego, si bien en principio, y como quizás el título ya ha sugerido a algún lector, parafraseamos a Spinoza, diciendo "la naturaleza, o la máquina", al modo en que él afirmó "Dios, o la naturaleza". En el caso spinozista, si la naturaleza es divina, el ser humano no tiene ninguna prioridad cosmológica; en este caso, si la naturaleza es ya técnica, el ser humano no tiene ninguna prioridad en medio de lo técnico.

Por otro lado, si bien estas hipótesis coinciden en observar lo técnico como carácter de la propia naturaleza, de ahí en más difieren. En este caso tomamos dos propuestas que nos parecen "ejemplares": la "ontología" maquínica en la obra conjunta de Deleuze y Guattari, y la ecotecnia propuesta por Jean-Luc Nancy. En el primer caso se da un paradigma de ensamblajes, mientras que en el segundo el autor insiste en un partes extra partes que subraya la imposibilidad de la conexión como tal, sin renunciar a cierta articulación de contigüidad. En fin, se juegan no solo dos ontologías de lo natural, sino, a su vez, conjuntamente, o una al lado de la otra, dos concepciones de la técnica. En síntesis, y en una jugada común de desnaturalización de la naturaleza, tanto una propuesta como la otra darán al caosmos, en los primeros, o multiverso, en el segundo, un carácter preminentemente técnico: la naturaleza no es otra cosa, anterior o más pura, que la técnica, sino un resultado de operaciones que cabría comprender como técnicas, desde allí, en la naturaleza y como naturaleza. Y sin embargo, no se trata en cada caso de la misma naturaleza ni de la misma técnica.

En primer lugar consideremos la propuesta esquizoanalítica de Deleuze y Guattari (2017), cuya puesta en operación se quiere antes ético-política que teorética, o todo a la vez; teor-ético-política. No obstante aquello, el principal recurso al que remiten sus esfuerzos es el de una hipótesis conectivista o de ensamblajes de la materia, hipótesis que se comprenderá como maquínica. De acuerdo a El AntiEdipo (2017) hay "[e]n todas partes máquinas, y no metafóricamente: máquinas de máquinas, con sus acoplamientos, sus conexiones. Una máquina-órgano empalma con una máquina-fuente: una de ellas 
emite un flujo que la otra corta" (Deleuze y Guattari 2017: 11), y esto opera desde el acople y ensamblaje entre la boca del bebé y el seno de su nodriza, hasta una comprensión del propio cuerpo -la boca, los ojos, los brazos, todos máquinas- $\mathrm{y}$, en fin, de toda la naturaleza, conceptualizada entonces como una interconexión y articulación de máquinas que conectan y transmiten flujos de una a otra, y que producen cortes en estos flujos. Estas máquinas son, también, las que sabemos funcionando en las fábricas, pero son más que eso: es la realidad misma, y su producción, la que debemos concebir maquínicamente, lo cual para estos autores -subrayamos- no es incurrir en metáforas. La actividad de estas máquinas es, sobre todo, productiva y las síntesis deseantes que estos autores propondrán no son sino modos de la producción maquínica de lo existente 5 . De aquí los autores concluyen que lo humano y la naturaleza convergen en tanto que actividades productivas maquínicas, sin por ello indiferenciarse:

"La esencia humana de la naturaleza y la esencia natural del hombre se identifican en la naturaleza como producción o industria [...] Hombre y naturaleza no son como dos términos uno frente al otro, incluso tomados en una relación de causa, de comprensión o de expresión (causa-efecto, sujeto-objeto, etc.). Son una misma y única realidad esencial del productor y del producto.” (Deleuze y Guattari 2017: p. 14).

Es la propia naturaleza la que opera como una industria, es decir, como una conjugación de máquinas, de modo que la producción y el producto no difieren, sino que hacen parte de una misma operación en curso. Esto no implica indistinguir absolutamente humanidad y naturaleza, sino anular la prioridad que una pueda tener sobre la otra, operando un giro en los conceptos que no cae en una indecisión nihilista: si hay aquí "relativismo" es solo en tanto la relación alcanza categoría de condición de realidad de lo existente, y no en tanto todo se vuelva indiferente.

En esta apuesta, en que Deleuze y Guattari (2017) siguen a Samuel Butler, no se trata ni de un vitalismo que invoque la necesidad de una unidad individual, orgánica y holista de lo vivo, excluyendo así a la máquina de esta relación de lo viviente al ser reducida a mecanismo y serie de efectos, y por tanto, separando a la máquina de los procesos deseantes; pero tampoco se trata de un mecanicismo que agote a

5El concepto de deseo operará como "principio inmanente" de las operaciones maquínicas, es decir, de los acoplamientos y cortes. Es una suerte de articulador insólito de las máquinas fragmentadas, que las pone en contacto, "hace fluir y corta". Aquí se pone al deseo no del lado de una falta a la que se tiende, sino del lado de los posibles que pueden desplegarse, es decir, el deseo da el carácter productivo a la ontología maquínica. En ese sentido, no busca tener connotaciones humanistas ni subjetivas, y en último término, se puede decir que la producción deseante es la que atañe a la propia naturaleza. 
la máquina en su definición estructural y atómica, individualizante al punto de dificultar pensar la unidad estructural a la que a máquina pertenece:

"El mecanicismo abstrae de las máquinas una unidad estructural según la cual explica el funcionamiento del organismo. El vitalismo invoca una unidad individual y específica de lo vivo, que toda máquina supone en tanto que se subordina a la persistencia orgánica y prolonga en el exterior sus formaciones autónomas." (Deleuze y Guattari2017: 293).

La propuesta de Deleuze y Guattari, a diferencia de un holismo vitalista o un atomismo mecanicista, invita a cambiar de registro para observar una microfísica de la producción deseante que es propiamente maquínica, es decir, de un empuje y despliegue de ensamblajes que lo componen todo. Allí habrá ocasión de distinguir "entre las máquinas molares por una parte, tanto si son sociales, técnicas u orgánicas, y las máquinas deseantes, que pertenecen al orden molecular, por otra parte" (Deleuze y Guattari 2017: 296). Esta distinción es ya una relación en tanto que ambas formas de la máquina, la molar-técnica y la deseante, son dos estados de la materia o, como dirían los autores, dos formas de segmentariedad, rígida la primera y más flexible la segunda. Pero ambos registros operan en relación. Así se logra huir a una determinación demasiado exacta de la técnica, que pudiera derivar en esa sutil tecnofobia comentada más arriba: las técnicas molares, como las tecnologías sociales, son soportadas por microprocesos moleculares, también maquínicos y productivos, que pueden transformar las cosas para hacerles devenir de otro modo. Así, queda una apertura para la tecnicidad, que ya no se constituye en sí misma como alienación: hay una suerte de ensamblaje naturaleza-historia o naturaleza-humano que se funda precisamente en lo maquínico, singular concepción de la técnica como operación inherente a lo existente, cuya consistencia es la productividad que emerge de las puestas en conexión de innumerables máquinas trabajando los flujos de lo real, y esto maquínico puede producir las tecnologías sociales que Stiegler asociaba a exteriorizaciones proletarizantes, pero no solo puede eso, de manera que se da lugar a una pregunta en torno a otras técnicas: al menos se disocia la alienación de la tecnicidad. Los autores propondrán que el funcionamiento deseante de las máquinas puede reprimirse, deviniendo alienante; sin embargo, también puede pasar la situación alternativa: las máquinas, sociales, técnicas y moleculares, todas, pueden insistir en su funcionamiento productivo liberado, creativo, insólito. 
Así, cierta inestabilidad propia del maquinismo, libera a la tecnicidad de ser inherentemente alienante, sin derivar en un optimismo tecnológico ciego, sino reconociendo la posibilidad de formaciones técnicas destructivas: hoy hemos llegado a un punto en que la indistinción técnica entre naturaleza y humanidad no promete mucho para ninguna de estas partes, y sin embargo, esto no se sigue de una necesidad de la técnica.

Pero, decíamos, a su manera también otra alternativa, menos conectivista, también apuesta por esta complicación, y en este caso tomamos en consideración el concepto de ecotecnia de Nancy. También se pone en juego una ontología de la relación, pero no ya de una relación conectiva, sino de una relación que subraya el entre como una distancia: cuerpos y, entre, espacio. Si se pudiera, diríamos que hay una suerte de atomismo en esta filosofía: los cuerpos se inclinan unos a otros, y en su aglomeración, componen mundos, y su aglomeración es, en cierto sentido -tomando un concepto althusseriano-, aleatoria. Pero no es el modo en que Nancy expresa la cuestión.

Nancy (2017) sostiene que los cuerpos son densos, impenetrables, que son "aparte", contiguos antes que continuos los unos de los otros, llenos - de otros cuerpos o llenos de sí mismos- extensos, a la vez que inmateriales, ya que también son un contorno, una organización o la forma de esa organización. Esto último, material pero invisible, recibe el nombre de alma, pero "[e]l cuerpo es un alma" (Nancy 2017: 17). Así, los cuerpos son fuerzas, diferencias, incluso respecto de sí mismos, pero nunca hay un cuerpo, siempre hay los cuerpos. Estos cuerpos pesan, se pesan unos a otros, declinan unos sobre otros, y así “componen", o articulan, un cuerpo cósmico, místico, "palmo a palmo, mi cuerpo toca todo [...] llego a las galaxias y finalmente a los límites sin fronteras del universo", "sustancia universal y marioneta tironeada por mil hilos" (Nancy 2017: 21). Esta colección de cuerpos no hace unidad, "no hay unidad sintética" del cuerpo, solo indicios de ellos (Nancy 2017: 27).

Así, vemos que el universo o multiverso, en tanto no se unifica, es un compuesto no totalizable ni sintetizable de cuerpos que, unos al lado de los otros, o encima o debajo, se pesan, se tocan, se contactan pero no se conectan, entran en contigüidad sin entrar en continuidad. Aquí, en este marco, tiene su lugar la técnica, pero concebida de un modo muy particular. Nancy (2006) verá la técnica no como un medio para un fin, sino como "un modo de ejecución, de manifestación y de actuación en general", "ser del ser" (Nancy 2006: 133). En esto no es distinta de la physis, también modo de acabamiento del ser, pero sí es 
otro acabado. Sin embargo no existe una contra la otra, sino que hay una torsión que afecta a esa diferencia y que Nancy situará como "la historia": es a partir de un momento que la técnica aparece como acabado del ser, caracterizada como finalidad sin fin extrínseco, pero radicalmente: sin fin. Nancy será quien spinozianamente afirme "Deus sive natura sive machina", que consideramos también conveniente para la hipótesis ontológica anterior, pero que en este caso busca dar de un proceso que no termina de terminar.

Profundicemos en ello: Nancy sostendrá que naturaleza y técnica no se oponen, sino que una llega a inscribirse en la otra en una torsión histórica, señalará "el hecho de que 'la técnica', que se añade a la 'naturaleza', y que abre fines que ella ignora, construye en realidad la idea misma de "naturaleza'" (Nancy 2013: 31), y a esto lo denominará "strucción". Desde aquí sintetizará:

Al hablar de 'naturaleza', suponíamos, o mejor dicho, superponíamos una coordinación propia e inmanente a la abundancia de seres (una construcción espontánea o bien divina). Con la 'técnica' suponíamos una coordinación reglada o regulada por los fines localizables a partir del 'hombre' (sus necesidades, sus capacidades, sus expectativas) [...] la 'técnica' confunde las dos posibilidades de coordinaciones. Invita a considerar una strucción. La simultaneidad no coordinada de las cosas o de los seres, la contingencia de sus copertenencias, la dispersión de las abundancias de aspectos, especies, fuerzas, formas, tensiones y distensiones (instintos, pulsiones, proyectos, impulsos). (Nancy 2013: 34).

Esta strucción es la "lógica" de la ecotecnia: aglomeración sin fines que hace mundo. Esta ecotecnia structiva aparecerá en Nancy (2006) como "tejne pura de la no-soberanía" (Nancy 2006: 148), “como fin in-finito", "existencia de lo existente finito, y su fulgor, y su violencia" (Nancy 2006: 153). Que este ser del ser corresponda a una torsión histórica se revela en el hecho de que no siempre fue así pensado, es decir, que sólo hoy llega a ser el ser del ser, hoy cuando el "mundo es el mundo de la técnica", el mundo del cual el cosmos, la naturaleza, los dioses, el sistema completo en su juntura íntima, se expone como «técnica»: mundo de una ecotecnia" creativa que crea, precisamente, la verdad de nuestro mundo de cuerpos (Nancy 2016).

Mientras en el conectivismo de ensamblajes la técnica era la operación productiva del deseo, en la ecotecnia ésta es la operación aglutinante de los cuerpos. En un caso subsiste un principio, si bien dislocado y no fijable, deseante, inmanente a su propio proceso. En el segundo caso se abre una brecha que invita al pensamiento, sin soltar hipótesis al respecto: des-orden, contingencia, fortuitidad, dispersión, 
enumera Nancy (2013). En ambos casos se logra disolver los diques que contenían a la naturaleza y a la técnica, para verlas abrazarse en un solo fenómeno que, también en ambas filosofías, aparece como propiamente sin un fin intrínseco ni extrínseco, sin telos.

Así, si bien en ambos casos hay una inestabilización de la diferencia naturaleza/técnica, lo que está en juego en cada caso son hipótesis "ontológicamente" distintas: en el caso de la fílosofía de Deleuze y Guattari, se trata de una inmanencia deseante, que opera flujos y cortes, conexiones, de manera que la naturaleza es una fábrica ensamblada de máquinas tramando y destramando flujos; mientras que, en caso de la filosofía de Nancy, se trata de una aglomeración contingente, sin modelo arquitectónico ni principio, con lo que la naturaleza es técnica o "artificial" precisamente por carecer de un empuje intrínseco, inherente a su "naturaleza". En el primer caso, lo técnico es lo maquínico como conectivismo productivo, deseante; mientras que, en el segundo caso, lo técnico es lo propiamente structivo.

En ambas filosofías, cada cual a su modo, se desplaza el lugar de lo humano o lo subjetivo. En la filosofía de Nancy este desplazamiento resulta de la deconstrucción del humanismo, de la noción de sujeto e incluso de la categoría del sentido. Estas son operaciones que se siguen de la propia desmesura de la historia humana, digamos, de su carácter structivo. Así, de cierta manera, el despliegue de la "potencia" humana es la que ha puesto en marcha su propia deconstrucción. Al revés, en Mil Mesetas (Deleuze y Guattari 2002), se comprenderá, en el marco de un continuo estratificado de la materia deseante, un estrato haloplástico que caracterizará a lo humano a partir de la técnica y la lingüística. Si bien no se cierra la posibilidad de hallar también allí, en ese estrato, a animales no humanos igualmente haloplásticos cuestión que dicha obra no abarca, pero, insistimos, no cierra- este estrato pareciera explicar más bien, más cabalmente, el "fenómeno" humano, esfuerzo ciertamente necesario. Sin embargo, esta opción redunda en establecer nuevamente, aún si más lábilmente, una distinción entre lo humano y el resto de la naturaleza.

De todos modos, cabe resaltar cómo, en ambos casos, para ambas filosofías, es una cuestión histórica la que tiene alcance ontológico: en el esquizoanálisis el capitalismo figura ya como un acontecimiento histórico-natural que modifica lo real, de manera que sus conceptos sirven al pensamiento del hoy, tanto como los esfuerzos de Nancy, cada cual a su modo. Así, un diferendo entre ambas propuestas parece jugarse a un nivel que escolásticamente podría denominarse de filosofía primera: es el enfrentamiento 
entre la inmanencia como tendencia de la materia, versus la contigüidad sin anulación de la distancia como tendencia de los cuerpos.

\section{Corolarios y excedentes}

Decíamos, al comenzar, que queríamos avanzar hacia una pregunta que modulara las categorías de lo natural, lo técnico y lo humano, hoy. Y las pistas de esta pregunta se hallan dispersas en esta, nuestra época: la técnica subordinada al humanismo y al capitalismo han transformado la naturaleza, haciendo que nos preguntemos: ¿es la técnica un carácter humano o bien lo propio humano? ¿Pero esto humano no tiene lugar en medio de una naturaleza, que hoy pareciera ser modificada o incluso destruida? ¿Cuál sería, entonces, la relación entre naturaleza y técnica? ¿No serán, también, lo mismo? ¿No se estará produciendo, monstruosamente, sea maquínica o structivamente, el mundo como natural? ¿No habrá sido siempre la naturaleza una entidad contranatura?

En primer lugar, queremos llamar la atención sobre la diferencia que aquí se ha abierto entre técnica y utilidad. Si la tecnicidad puede pensarse como el ser de la materia, esta ha de observarse antes como acontecimiento o proceso, y no como estrategia, medio para un fin o utilidad. Como mencionamos antes, si Stiegler puede ver en la técnica procesos de hominización, quisiéramos recordar que también otros animales no humanos usan diversos recursos técnicos, eficientes respecto de su vida en sus entornos, no al modo de la utilidad humanista, sino de la composición orgánica o ecosistémica. Por eso aquí no nos detenemos en la Ge-stell heideggeriana (2019): si bien es históricamente cierto que la técnica, en el marco del capitalismo, se ha convertido en un dispositivo monstruoso que reduce el ser de lo existente a la figura empobrecida del recurso a disposición de su explotación y acumulación, esto da cuenta del carácter del capitalismo antes que de la tecnicidad. En otras palabras, pensamos que la técnica da muestras de ser un fenómeno histórica y naturalmente más vasto que aquello que no es sino la técnica en el marco del capital: ¿acaso tendríamos que ver en las técnicas del éxtasis, estudiadas por Eliade (2009), o en las herramientas de los chimpancés, estudiadas por la etología, o en la alquimia medieval, actividades que merecerían otros nombres, para conceder que la técnica solo es, intrínsecamente, la disposición tendencialmente destructiva de los entornos ecológicos, del resto de las especies y de la humanidad misma? Si se nos permite tomar prestada la noción de ecosofía guattariana, diremos que además del cuidado, producción y proliferación de especies sociales, mentales y ambientales, hoy está en juego también la consideración de especies técnicas, y aquí el recurso a la historia, la antropología o la etología no busca caer en ningún sesgo utópico 
ni primitivista: ¿por qué pensar la técnica en la clave del mal menor respecto de la acumulación de capital, pudiendo pensarla como una composición maquínica o structiva, presente en variadas especies, que incluso promueva el enriquecimiento de ecosistemas ambientales, mentales y sociales?

Con total justeza se podrá argüir que quizás eso ya ocurre, y que no cabe observar desde perspectivas recargadas de juicios morales la actual situación del mundo: la técnica de la Ge-stell ha dado su hábitat, gris y urbano, a ratas, palomas, bacterias, mohos, moscas, perros callejeros, y otros vivientes. ¡La ecotecnia ha hecho mundo y los nostálgicos no lo quieren ver! Pues respondemos que eso es lo que está en juego, y por evaluarse tras ser articulada una pregunta: así, Nancy se pregunta por la hipótesis de una destinerrancia que nos queda, y que nombra también como el sentido de la errancia del sentido; mientras, a su modo, Deleuze y Guattari permiten plantear la pregunta por el carácter deseante o reprimido de las máquinas que producen este mundo; y, finalmente, Stiegler pone en primer plano el empobrecimiento humano que la actual forma de vida puede llegar o está promoviendo, proponiendo una terapéutica alternativa a la alienación que diagnostica en curso. Destilamos de estas tres propuestas una interrogante que, desde una coincidencia entre técnica y naturaleza, se hace una pregunta a la vez ontológica y, si cabe, caosmopoiética, intentémoslo: ¿el fin del humanismo, y por ende de su técnica contrapuesta a la naturaleza, coincidirá con la clausura de todos los posibles, con la sofocación, o puede desatar fuerzas creativas, simbióticas y sinérgicas, trans-técnicas, trans-naturales, trans-humanas: ventilar este ahogo?

Intentémoslo otra vez, mejor. Y es que acaso una pregunta que interrogue nuestro hoy para disparar otras hipótesis pueda plantearse a partir de todos estos tanteos y revisiones: si la naturaleza se ha revelado técnica ella misma, y el ser humano, sin ninguna centralidad ontológica a este respecto, ha operado ciertamente como bisagra de este proceso o como su aspecto más notorio, ¿cómo se hace mundo de aquí en más, cuando esta cuestión ya no es ética y la política se ve obligada a replantearse, cuando lo que el "mundo" pone en juego es su propia composición técnica, maquínica, structiva y/o errante, de cuerpos, materia, flujos y/o cortes, cuando la historia se ha revelado natural, que se ha revelado cósmica, que se ha revelado artificial o artefactual?

La modulación de estos caracteres no pretende abrir una pregunta hacia el futuro, sino más bien hacia una comprensión de este presente esquivo que parece no contar con categorías que le sean propias, 
comprensión que nos urge hoy y que solo asume la formulación de preguntas, eludiendo (todavía) una respuesta. El hoy, como pregunta ¿lo ven, lo oyen?

Referencias bibliográficas:

Deleuze G. \& Guattari F. (2002), Mil mesetas: Capitalismo y esquizofrenia (5. ed), Valencia, Pre-Textos. (2017), El Anti-Edipo: Capitalismo y Esquizofrenia, Buenos Aires, Paidós.

Derrida J. (1997), La Diseminación. Madrid, Editorial Fundamentos.

Eliade M. (2009), El chamanismo y las técnicas arcaicas del éxtasis, México, Fondo de Cultura Económica.

Fisher M. (2019), K-Punk-Volumen 1. Escritos reunidos y inéditos (Libros, películas y televisión),Buenos Aires, Caja Negra Editora.

Guattari F. (1996), Las Tres Ecologías, Valencia, Pre-Textos.

Haraway D. (2016), Antropoceno, Capitaloceno, Plantacionoceno, Chthuluceno: Generando relaciones de parentesco, Revista Latinoamericana de Estudios Críticos Animales, III, vol. I, 15-26.

Heidegger M. (2019), Filosofia, Ciencia y Técnica, Santiago, Editorial Universitaria.

Nancy J. L. (2006), Ser Singular-Plural, Madrid, Arena Libros.

(2007), 58 indicios sobre el cuerpo: Extensión del alma, Buenos Aires, La Cebra.

(2013), Archivida. Del sintiente y del sentido, Buenos Aires, Quadrata.

(2016), Corpus, Madrid, Arena.

Simondon, G. (2007), El modo de existencia de los objetos técnicos, Buenos Aires, Prometeo Libros.

Cactus.

(2015), La individuación: A la luz de las nociones de forma y de información, Buenos Aires,

Stiegler, B. (1994), La Técnica y el Tiempo 1. El pecado de Epimeteo, Madrid, Cultura Libre.

$\overline{6,133-146 .}$

(2012), "Tiempo e individuaciones técnica, psíquica y colectiva”, en Simondon. Trilogía, No.

(2014), "Ars Industrialis e Invenciones organológicas en las sociedades de hiper control", Revista de Filosofía No. 28, 147-163. 
(2016), Para una nueva crítica de la economía política: Sobre la miseria simbólica y el complejo económico-político del consumo, Buenos Aires, Capital Intelectual. 